\title{
Development on Construction Project Cost System Based on Spring
}

\author{
Nannan Zhang ${ }^{1, a}$, Wanqing Yang ${ }^{2, b}$ \\ ${ }^{1}$ School of Management, Bohai University, Jinzhou, 121013, China \\ ${ }^{2}$ State Grid Dalian Electric Power Supply Company, Dalian, 116001, China \\ a331263907@qq.com, bywq13998650041@sina.com
}

\begin{abstract}
Keywords: Spring; processing flow; construction project; cost system; development; system functional design; data access design
\end{abstract}

\begin{abstract}
Spring is to solve the common problem in many J2EE development, and can replace strong lightweight framework in the EJB technology. Based on Spring development construction project cost system, to improve the competitiveness of building enterprise and information technology services. First of all, based on the Spring framework structure, the Spring MVC request processing process; Then, following the abstract and incremental refinement, the principle of modularity and information hiding, designed by the six main function modules of the system; Finally, using the JDBC method to design the data access, including entity class structure and write two steps to implement the DAO. The research achievements of this paper to solve the key technical problems in the software development process, the actual development process to exert the advantages of Spring, to avoid the shortcomings, in order to improve the software performance.
\end{abstract}

\section{Introduction}

Throughout the whole process of the project, the project cost management is an important part of project management. Engineering cost management object is the construction project, take the way of management in engineering construction activities, in order to improve the economic benefit as the goal, to project cost planning, organization, coordination, control and management. Current construction engineering cost management are widespread lack of authenticity, difficult to material price estimate, restricted by many problems, such as management department. Construction project cost system developed by the network and database technology, the construction project cost calculation analysis and evaluation of each link, provide auxiliary support for the leadership decision-making, for engineering and equipment material information transmission, storage and maintenance. At the same time, the information of multiple departments, realize data sharing and mutual restriction, effectively solve the caused by management problems in the construction project cost control.

The MVC (Model View Controller) is a software design Model, using a method of separation of business logic, data, interface display organization code, the business logic gathered on a widget, in improving and personalized custom interface and user interaction at the same time, do not need to write the business logic. The Spring is open source of the MVC framework, is to solve the complexity of enterprise application development and create [1]. Spring is to solve the common problem in many J2EE development, and can replace the EJB technology strong lightweight framework. Spring of light is embodied in its structure, the basis of the framework itself, as well as support for other applications and assembly capacity. Compared with EJB this Spring can make the application developers to lower risk between the various technical level. Spring is not only confined to the server-side development, from simplicity, testability and loose coupling point of view, any Java application can use the Spring. In this paper, based on the Spring development construction project cost system.

\section{Spring Process}

Spring uses the set value of injection and structure, the mechanism of injection by configuration file management to form a collaboration object, create structural components of the IoC container. The 
Spring framework is composed of seven well-defined modules, and each module or component can exist alone, or with other one or more modules. "The Spring Core Container" used to manage the business component of the IoC Container, is the Core of Spring applications. "Spring DAO" and "Spring ORM" not only provides a data access abstraction module, are integrated to Hibernate, JDO and iBatis popular object-relational mapping framework support module, and provides a buffer connection pooling, transaction processing and other important services function, ensure that the system performance and data integrity; Sprnig Web module provides a Web application to some abstract encapsulation, Struts, WebWork, etc can be Web framework with Spring integration is suitable for their own solutions. The Spring framework structure as shown in Fig.1 [2].

\begin{tabular}{|c|c|c|c|}
\hline \multirow{2}{*}{$\begin{array}{c}\text { Spring } \\
\text { AOP }\end{array}$} & Spring ORM & Spring Web & \multirow{2}{*}{$\begin{array}{l}\text { Spring } \\
\text { MVC }\end{array}$} \\
& Spring DAO & Spring Context & \\
\hline \multicolumn{2}{|c|}{ Spring Core Container } \\
\hline \multicolumn{2}{|c|}{} &
\end{tabular}

Fig. 1. Constituent on Framework Module of Spring

Spring MVC design revolves around DispatcherServlet, the DispatcherServlet is responsible for the request sent to a specific handler. Through configurable handler mappings, view resolution, locale and theme resolution to handle requests and transferred to the corresponding view. Spring MVC request processing process is shown in Fig. 2 [3].

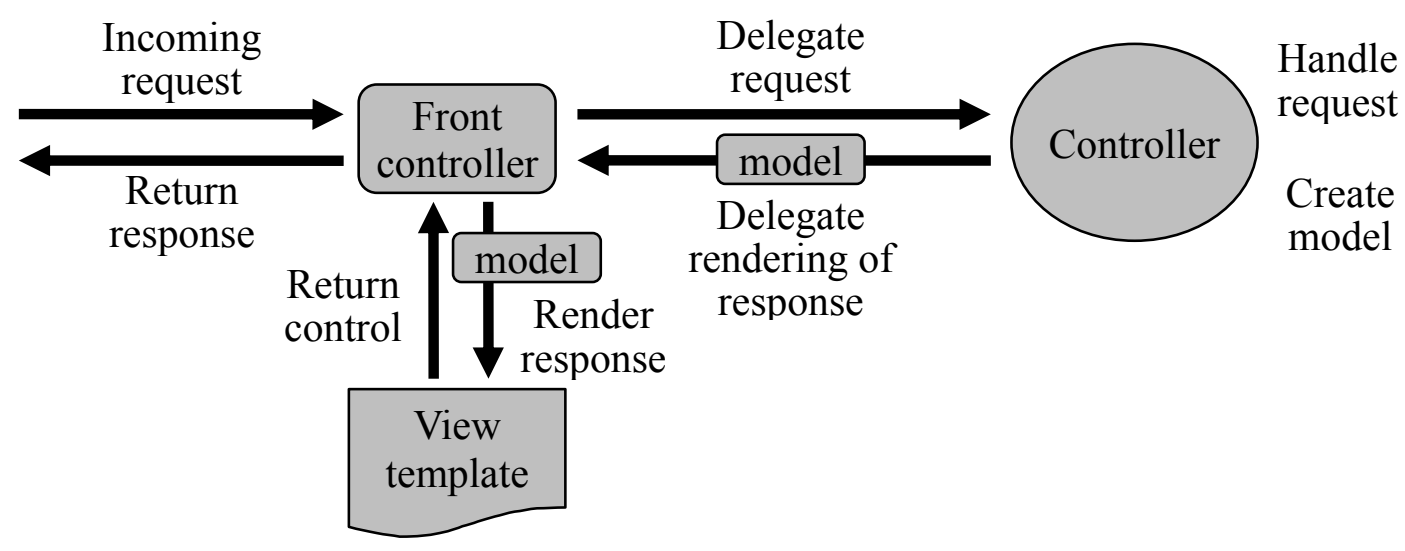

Fig. 2. Request processing flow of Spring

(1) When the DispatcherServlet received a request to find out the appropriate handler to handle the request. DispatcherServlet via one or more handlers mapping, to map each request to the handler. Handler mapping configuration in the context of web applications, is implements the interface HandlerMapping Bean, responsible for the request to return to an appropriate handler (the Controller). Usually according to the request URL will be mapped to the handler [4];

(2) The DispatcherServlet chose the appropriate controller, the controller will call to handle the request;

(3)After processing the request, the controller object model and the view will be returned to the DispatcherServlet. Model contains a controller to pass to the view for display properties. If return to view name, will be parsed into view object for rendering. The basic class is ModelAndView of binding model and view;

(4) When the DispatcherServlet receives the model and view name, logical view name resolution into view object for rendering. DispatcherServlet from one or more views the view that the parsed by a parser. View parser configuration in the Web application context, is to implement the interface ViewResolver Bean, task is according to the logical view name return view object;

(5) The DispatcherServlet will view name resolution called view object, will present the view object, and return transfer controller model. View of the task is to show to the user model properties. 


\section{System Functional Design}

Functional design should follow the abstraction, incremental refinement, the principle of modularity and information hiding, etc. Abstraction is the concentration and sum up the similar aspects, ignored the differences between them; Stepwise refinement is a kind of top-down design strategy, the software architecture, according to the top-down approach, details and data details the process of all levels of refinement gradually, until can statements in a programming language to realize, finally established the whole software architecture; Information hiding is refers to the implementation details of each module is hidden for other modules, information contained in a module, don't allow other don't need these information module USES; Effective modularity can be realized by defining a set of independent modules, these modules communication between each other just use for implementing software function is the necessary information. The results of the functional design is through the hierarchy chart to describe the software structure. Hierarchy chart of a rectangular box represents a module, a rectangular box attachment represent the relationship between, located at the top of the rectangular box module, call in at the bottom of the rectangular box on behalf of the module. This system function design only two layer structure, as shown in Fig.3.

\begin{tabular}{|c|c|}
\hline \multicolumn{2}{|c|}{ Construction project cost system } \\
\hline Template information management & \\
\hline Supplier information management & Project cost management \\
\hline Cost model management & Cost control management \\
\hline
\end{tabular}

Fig. 3. Function on construction project cost system

In the function design results shown in Fig.3, including six modules, briefly describe the following [5-7] :

(1) The template information management. According to the features of construction engineering project design the corresponding project cost template. Function requirement to be able to build the cost template, template information query, modify the template, delete the template and the template parameter Settings;

(2) Supplier information management. In the new building materials procurement situation, puts forward the management mechanism, is the key link in supply chain procurement management, in the implementation just-in-time purchasing has a very important role. Function requirements of the supplier information, product information, quotation information and management;

(3) The cost model of management. Model means that for some actual problem or objective things, the law after the abstraction of a formal expression. Project cost estimation results according to the input data calculated by the model, and the need to provide to add, modify, delete the model, and other functions;

(4) The Project cost management. Is the core function of this system, used for architectural engineering cost fee structure and the cost of the raw data management;

(5) The Cost control management. Control management refers to the business activities of inspection and supervision, to determine the progress of the activity, the actual work and the work plan of correcting deviation, thus ensuring the whole plan and the realization of organizational goals.

(6)The System maintenance management. For the function of the system administrator to provide, in order to ensure the normal operation of the system, and can deal with system environment become public, of information maintenance and system management, etc. 


\section{Data Access Design}

JDBC (Java Data Base Connectivity) is a Java API for executing SQL statements for a variety of relational database data access method. Spring is an open source framework, the core technology is a lightweight container, based on the J2EE enterprise application development application framework to provide comprehensive support to [8]. Based on Spring framework to develop a Java application, there are two kinds of data access methods, one is Spring JDBC, the other is a Spring Hibernate. Based on the "schedule of project cost" as an example, the design using the Spring JDBC data access. "Schedule of project cost" logic structure as shown inTable 1.

Table 1. Project costs detail table

\begin{tabular}{c|l|l|c|c}
\hline No & Fields Name & Type & Width & Fields explain \\
\hline 1 & IDzgjz & Integer & 4 & Primary key identity \\
\hline 2 & XBXXxmbm & Char & 8 & Project encoding \\
\hline 3 & FYMCfybm & Char & 4 & Costs encoding \\
\hline 4 & XMZJfyje & Decimal & 10,2 & Costs amount \\
\hline 5 & XMZJysrybm & Varchar & 50 & Budget personnel encoding \\
\hline 6 & XMZJysrqsj & Timestamp & 7 & Budget date time \\
\hline 7 & XMZJysyj & Long Varchar & 1000 & Budget basis \\
\hline
\end{tabular}

Spring JDBC provides some methods and corresponding different classes interact with the database. The most classic and popular is the use of JdbcTemplateClass framework. JdbcTemplate execute an SQL query, update statement and stored procedure call, in the result set and extracting return parameter value iteration. Also capture the JDBC exception and transform it into universal, more abundant information, in the org.Springframework. The dao package defined in the hierarchy. JdbcTemplate instance of a class is a configuration of threads. You can configure a JdbcTemplate an instance, and then safely into the Shared references to multiple DAO. Using JdbcTemplate class, the usual approach is to configure a DataSource in the Spring configuration file, then the Shared data sources to the DAO class dependency injection, JdbcTemplate, or in the setter data source creation. Complete data access is mainly in two steps.

Step 1: Entity class structure. Entity class is commonly known as POJO, mainly as a data management and business logic processing level category, this class is not implemented within the framework of the special interface, just as a data container used to store data persistence. Entity class code is as follows:

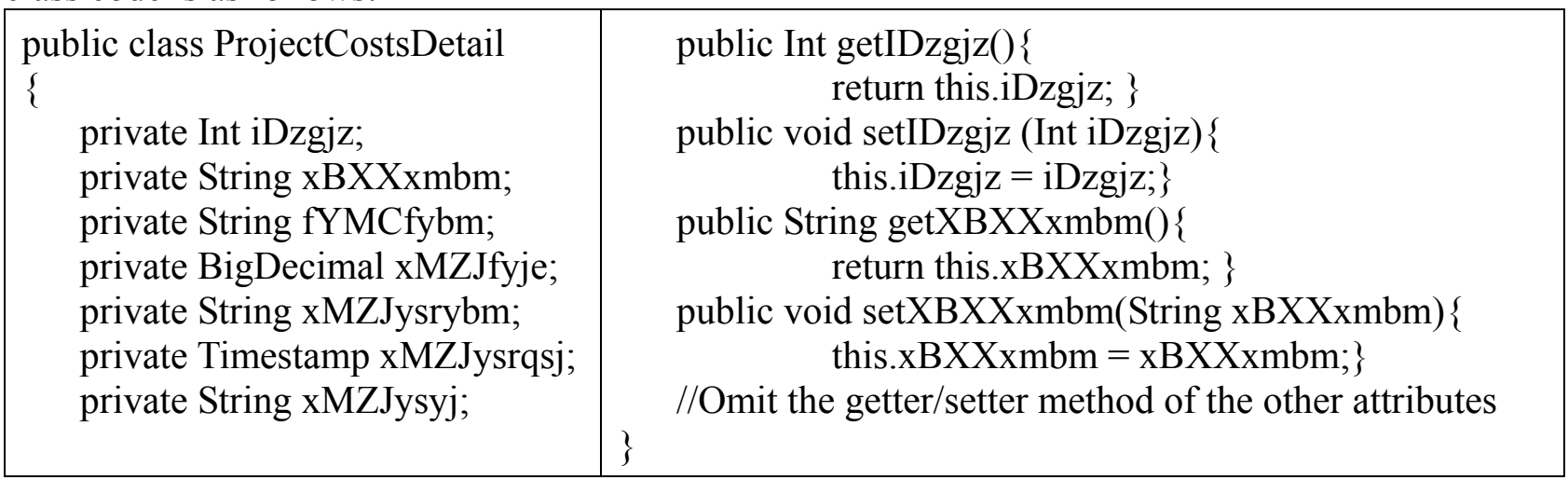

Step 2: Write the DAO implementation. The Data Access Object (DAO) is one of the J2EE design patterns, the low-level Data Access logic from high-level business logic. Spring JDBC DAO inherits from Spring to provide JdbcDaoSupport classes. Spring brought benefits to the writing of the DAO, refine the data access exception, enrich the exception type; Using templates callback mode, mda developers write code, to simplify programming. Based on JDBC DAO implementation, simplify the operation of JDBC, JDBC objects for release, exception type conversion; Developer is 
responsible for providing SQL, set the variables in the SQL, extracting the ResultSet. Limited to space, the DAO implementation include a getHemobiologicalIndices class methods, only by calling this method can get all the field information. The DAO implementation code is as follows:

\begin{tabular}{|c|c|}
\hline $\begin{array}{l}\text { package cn.wode.springcore.jdbc; } \\
\text { import java.sql.ResultSet } \\
\text { import java.sql.SQLException; } \\
\text { import java.util.List; } \\
\text { import javax.sql.DataSource; } \\
\text { import org.springframework.jdbc.core.RowMapper; } \\
\text { import } \\
\text { org.springframework.jdbc.core.support.JdbcDao } \\
\text { Support; } \\
\text { public class ProjectCostsDetailDAO extends } \\
\text { JdbcDaoSupport implements } \\
\text { IProjectCostsDetailDAO } \\
\text { public List getProjectCostsDetail() \{ } \\
\text { String sql="SELECT iDzgjz, xBXXxmbm, } \\
\text { fYMCfybm, xMZJfyje, xMZJysrybm, } \\
\text { xMZJysrqsj, xMZJysyj FROM } \\
\text { projectCostsDetail"; }\end{array}$ & $\begin{array}{l}\text { return super.getJdbcTemplate().query (sql, new } \\
\text { ProjectCostsDetailRowMapper()); \} } \\
\text { protected class HemobiologicalIndicesRowMapper } \\
\text { implements RowMapper \{ } \\
\text { public Object mapRow(ResultSet rs, int rowNum) } \\
\text { throws SQLException \{ } \\
\text { Item item = new Item(); } \\
\text { item.setIDzgjz(rs.getInt("iDzgjz")); } \\
\text { item.setXBXXxmbm(rs.getString("xBXXxmbm")); } \\
\text { item.setFYMCfybm(rs.getString("fYMCfybm")); } \\
\text { item.setXMZJfyje(rs.getBigDecimal("xMZJfyje")); } \\
\text { item.setXMZJysrybm(rs.getString("xMZJysrybm")); } \\
\text { item.setXMZJysrqsj(rs.getTimestamp("xMZJysrqsj")); } \\
\text { item.setXMZJysyj(rs.getString("xMZJysyj")); } \\
\text { return item;\} } \\
\text { \} }\end{array}$ \\
\hline
\end{tabular}

\section{Conclusion}

The Spring framework has the following advantages [9] : lightweight container framework with no intrusive; Using a combination of the IoC container easier to direct relationships between objects, programming to an interface, reduce the coupling; Aop can be more easier for function extension, follow the principle of OCP development; Create an object by default is a singleton, no longer need to use the singleton pattern for processing. The disadvantage of the Spring framework is a function of business functions depend on the Spring characteristic, depend on the environment, and the Spring. Construction project cost system is not only an information management system, at the same time also reflect scientific and standardized management system and management system. System application not only better control, reduce the investment cost, but also allows the various departments to coordinated and Shared data resources, in the control of progress and quality at the same time, ensure the reasonable control of engineering cost, truly realized how fast "save" project construction goal. In the development process to exert the advantages of Spring, to avoid the shortcomings, in order to improve software performance.

\section{References}

[1] Seth Ladd, Darren Davison, Steven Devijver and Colin Yates, "Spring MVC Application Architecture," Springer eBook, 2016-01-22.

[2] IBMdeveloperWorks, "Spring series: introduction to the Spring framework," http://www.ibm.com/developerworks/cn/java/wa-spring1/, 2014-2-1.

$$
\text { wawlian' Blog, "MVC Spring }
$$

http://www.cnblogs.com/wawlian/archive/2012/11/17/2775435.html, 2016-2-2.

profile,"

[4] kobejayandy' special column, "Request process and source analysis of Spring MVC," http://blog.csdn.net/kobejayandy/article/details/12585745, 2015-1-27.

[5] Krzysztof Zima, "The Case-based Reasoning Model of Cost Estimation at the Preliminary Stage of a Construction Project," Procedia Engineering, vol. 122, pp. 56-57, 2015.

[6] X. K. Wang, Y. B. Zhou, "Analysis of construction project cost project management information system," Journal of Yantai Vocational College, vol. 22, no. 2, pp. 90-92, 2015. 
[7] L. L. $\mathrm{Xu}$, "Research on design and implementation of construction cost management information system," Information \& Communications, vol. 29, no. 9, pp. 117-118, 2015.

[8] J. Du, L. J. He, H. J. Ouyang "Design and Implementation of Online Examination System Based on JDBC and DAO Mode," Journal of Xianyang Normal University, vol. 29, no. 2, pp. 26-29, 2014.

[9] lishuangzhe7047' Blog, "Spring framework learning," http://blog.csdn.net/lishuangzhe7047/article/details/20740209, 2016-2-2. 